\title{
A new significance on the vertical component ratio of the power spectra between two sites in the application of array methods
}

\author{
Xinrui Zhang • Hitoshi Morikawa
}

Received: 29 December 2014 / Accepted: 12 August 2015 / Published online: 5 September 2015

(C) Springer Science+Business Media Dordrecht 2015

\begin{abstract}
Array methods like spatial auto-correlation (SPAC) method and the centerless circular array (CCA) method have provided a convenient means of inferring the phase velocity of surface waves. However, these methods are under the assumption of horizontally layered medium (lateral homogeneity) while the ground structure is actually likely to be inclined. Hence, it is expected to obtain more detailed information of ground structure such as inclination by making better use of the records. In recent years, the seismic interferometry theory has also been widely used to estimate ground structure. According to seismic interferometry theory, the cross correlation of motion between two sites is proportional to the imaginary part of the Green's function (IOG) between the two sites in diffuse wavefield. In this study, we can obtain the ratio of IOG between two sites by taking the ratio of power spectra between the same two sites. We propose this ratio as an indicator of the lateral heterogeneity between two sites. Through numerical simulation and a field test, we demonstrate that the significance of ratio of power spectra can be interpreted from the sight of ratio of IOG successfully.
\end{abstract}

\footnotetext{
X. Zhang $(\bowtie) \cdot$ H. Morikawa

Department of Built Environment, Tokyo Institute

of Technology, W8-22, 2-12-1, Ookayama, Meguro-ku,

Tokyo, 152-8552, Japan

e-mail: ricky.xinruizhang@gmail.com

H. Morikawa

e-mail: morika@enveng.titech.ac.jp
}

Keywords Array method-Seismic interferometry · Ratio of imaginary part of Green's function . Lateral heterogeneity $\cdot$ Spectral ratio

\section{Introduction}

Since Aki (1957) proposed a new approach to estimate phase velocities of surface waves, spatial autocorrelation (SPAC) method has been a very useful tool to estimate ground structure because of its simple post-process (Aki 1957). After that, many researchers both in and out of Japan continued to publish papers to extend Aki's theory and developed a variety of array methods (Okada 2003; Morikawa et al. 2004; Cho et al. 2006; Chávez-García et al. 2005, 2007; Shiraishi et al. 2006; Tada et al. 2009). However, all those improved methods are based on the assumption that the layers under surface are horizontal. In other words, the lateral heterogeneity have not been taken into consideration by the array methods above. However, the layers are likely to be inclined in real cases and it is expected to obtain more detailed information of ground structure such as inclination by making more use of the records from multiple sites in an array.

In this article, we use "horizontally layered structure," which actually means homogeneous structure, to distinguish from "layered medium with lateral heterogeneity," which means the inhomogeneous 
structure. Each layer of medium has the same property including density, S-wave velocity, and P-wave velocity.

In recent years, the seismic interferometry theory has also been popular in estimating ground structure. The Green's function between two points $\mathrm{A}$ and $\mathrm{B}$ means the response at $\mathrm{A}$ when a unit impulse force is exerted at $\mathrm{B}$ in an actual medium. Claerbout (1968) showed that the autocorrelation of the transmission response of an arbitrary horizontally layered lossless earth yields its reflection response in 1D model. This conclusion was generalized to three-dimensional inhomogeneous media (Wapenaar et al. 2002, 2004). Similar conclusions have been reached from much research in recent years (Weaver and Lobkis 2001; Campillo and Paul 2003; Wapenaar 2004; Wapenaar et al. 2006; Wapenaar and Fokkema 2006; Sánchez-Sesma et al. 2006; SánchezSesma et al. 2008; Snieder 2004; Perton et al. 2009). These theories are simply summarized as seismic interferometry (SI) theory.

It is demonstrated that in an elastic medium, the Fourier transform of azimuthal average of the cross correlation of motion between two sites is proportional to the imaginary part of the Green's function (IOG) between the two sites in frequency domain (Sánchez-Sesma and Campillo 2006; Sánchez-Sesma et al. 2008; Campillo and Paul 2003). Accordingly, it becomes possible to calculate the ratio of imaginary part of different Green's function by taking the ratio of corresponding cross correlation to analyze ground structure more particularly because Green's function indicates intrinsic property of the medium.

The horizontal-to-vertical spectral ratio $(\mathrm{H} / \mathrm{V})$ method has been shown to have strong relationship with the ratio of IOG in the horizontal and vertical direction in diffuse wavefield (Sánchez-Sesma 2011; Kawase et al. 2011). The peak frequency has been confirmed to match with each other in their articles. We can say that the H/V spectral ratio is successfully reinterpreted and becomes more revealing using seismic interferometry theory. Moreover, seismic interferometry is consistent with the SPAC method (Yokoi and Margaryan 2008; Tsai and Moschetti 2010; Paul et al. 2005) in diffuse wavefield, which forms the base of using seismic interferometry theory to array methods based on the SPAC method. Then, it is available to just apply $\mathrm{H} / \mathrm{V}$ spectral ratio method to each site in array methods to distinguish the difference of ground structure among all observation sites.

On the other hand, we consider using spectral ratio of only vertical component between two sites to detect the lateral heterogeneity. There are two advantages compared with $\mathrm{H} / \mathrm{V}$ spectral ratio method: first, only vertical component is used in both array method and the calculation of spectral ratio, which is more convenient for observation and data analysis; secondary, the vertical spectral ratio taken between two sites has the potential to demonstrate the lateral heterogeneity more clearly and directly than taking $\mathrm{H} / \mathrm{V}$ spectral ratio site to site and doing comparison. According to seismic interferometry theory, the spectral ratio taken between two sites is equivalent to the corresponding ratio of IOG. IOG is independent of the wavefield and can be calculated out as long as the ground structure between two sites are known (Hisada 1994, 1995). Thus, inversely, by knowing the vertical component spectral ratio, the difference of ground structure between two sites, namely the lateral heterogeneity, is likely to be detected.

It must be claimed that the application of spectral ratio between two sites is not a new idea. At first, the Fourier amplitude spectra of horizontal components at a site has been used to identify the resonance peak of the soil layer (Kanai and Tanaka 1954). Afterwards, the method to apply spectral ratio between two sites has also been developed decades ago (Kagami et al. 1986). If one site is on the rock (reference site) and one is on soil, by taking the spectral ratio between them, the resonance of the soil layer can be detected. However, there are three disadvantages of this theory: first, this method is based on the assumption that the motion at the reference site should be representative of the excitation arriving at the interface of soil layer and base (Lermo et al. 1994); second, this method confines the two sites to be on the soil and on the rock, which lacks the generality to detect the lateral heterogeneity; third, this theory is based on the assumption of 1D ground structure. Moreover, horizontal components are usually used in this method. In this study, instead, we regard vertical component spectral ratio between two sites as the ratio of imaginary part of Green's function. The wavefield just needs to be diffuse and the two sites have no limitations. It not only gives a new perspective of the spectral ratio between two sites but also becomes a general indicator of lateral heterogeneity. 
In this article, first we will introduce the theoretical background and propose the new perspective of vertical component spectral ratio between two sites. For convenience, we use the ratio of power spectra to replace spectral ratio (ratio of Fourier spectra). Secondary, we will use a simple inclined two-layered model based on finite difference method (FDM) (Pitarka 1999) to discuss how the vertical component ratio of IOG between two sites will behave under simple lateral heterogeneity. Next, we use FDM to create a diffuse wavefield in the same model. In this wavefield, we calculate the vertical component spectral ratio among sites in a linear array and examine whether they coincide with the theoretical ratio of IOG, respectively. Finally, a field test, in which the information of ground structure is given, is used to confirm the availability of the theory.

\section{Theoretical background}

In this chapter, the basic knowledge of array methods and seismic interferometry will be introduced and our new concept of combining them two will be proposed.

\subsection{A simple introduction of array methods}

For simplicity, we introduced the typical array method, SPAC method here. Given an equilateraltriangle array and vertical time series records of microtremors at four sites under the assumption of temporally and spatially stationary wavefield, the azimuthal-average of spatial auto-correlation coefficients $\rho\left(\frac{r \omega}{c(\omega)}\right)$ can be calculated as:

$\rho\left(\frac{r \omega}{c(\omega)}\right)=\frac{1}{3} \sum_{j=1}^{3} \frac{S_{0 j}(\omega)}{\sqrt{S_{00}(\omega) S_{j j}(\omega)}}$,

where $\omega, r$, and $c(\omega)$ are the angular frequency, radius of the circular array, and phase velocity, respectively. $S_{j j}(\omega)$ and $S_{0 j}(\omega)$ are the power spectra of vertical component of microtremors at site $j(j=0,1,2,3)$ and the cross spectra of the vertical records between site $j$ and 0 . The site 0 is center of the array and sites 1, 2, and 3 are located on the circle as shown in Fig. 1.

Practically, $S_{j k}(\omega)$ is obtained by the product of Fourier transformation $F\left(\omega, X_{j}\right)$ of records at two sites as

$S_{j k}(\omega)=F\left(\omega, X_{j}\right) F^{*}\left(\omega, X_{k}\right)$,
Fig. 1 Geometry used in conventional SPAC method

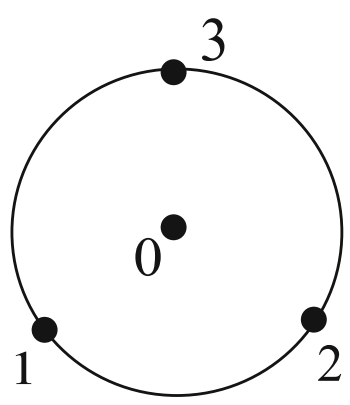

where $X_{j}$ is a location of site $j$ and * stands for the complex conjugate.

Afterwards, a dispersion curve can be obtained and the property of under-surface layers are estimated using certain inversion technique.

\subsection{Seismic interferometry}

It has been demonstrated (Sánchez-Sesma and Campillo 2006) that in an elastic medium in the diffuse wavefield satisfying the energy equipartition, the averaged cross spectra of motions at sites 1 and 2, whose locations are $X_{1}$ and $X_{2}$, can be written as:

$$
\begin{aligned}
& <F_{\ell}\left(\omega, X_{1}\right) F_{m}^{*}\left(\omega, X_{2}\right)> \\
& =-2 \pi E_{s} k^{-3} \Im\left[G_{\ell m}\left(X_{1}, X_{2}, \omega\right)\right],
\end{aligned}
$$

where $\ell$ and $m$ indicate one of three directions, $(\mathrm{x}, \mathrm{y}, \mathrm{z})$. $k$ and $E_{s}$ are wave number of shear wave and the averaged energy density of shear wave, respectively. $<\cdot>$ stands for an expectation and $\Im[\cdot]$ for the imaginary part.

\subsection{A new perspective of ratio of power spectra between two sites}

Now, we constitute a vertical component ratio of power spectra through Green's function. Both of $\ell$ and $m$ are set as vertical direction, which is $z$, and sites 1 and 2 as the same sites. Hence, it becomes

$$
\begin{aligned}
< & F_{z}\left(\omega, X_{1}\right) F_{z}^{*}\left(\omega, X_{1}\right)>=-2 \pi E_{s} k^{-3} \mathfrak{s} \\
& {\left[G_{z z}\left(X_{1}, X_{1}, \omega\right)\right] }
\end{aligned}
$$

The left-hand side becomes the power spectra at one site and according to the right-hand side, the power spectra contains information about both the strength of the wavefield $\left(E_{S}\right)$ and intrinsic property of ground structure $\left(\Im\left[G_{z z}\left(X_{1}, X_{1}, \omega\right)\right]\right)$. Now, it is required to extract out only the required part which is 
Fig. 2 The profile of the simple horizontally two-layered medium for testing the validity of the code based on FDM

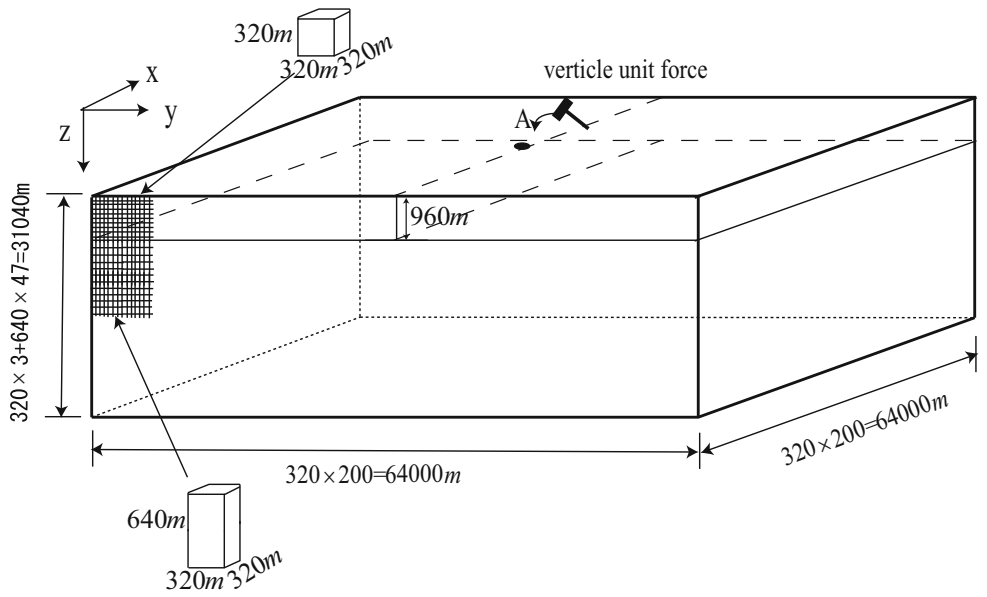

the Green's function and remove unknown values of parameters such as $E_{s} k^{-3}$. Hence, we take the ratio of the power spectra at center of the array and a site on the circle. As a result, the corresponding ratio of IOG can be obtained as

$\frac{S_{j j}(\omega)}{S_{00}(\omega)}=\frac{\Im\left[G_{z z}\left(X_{j}, X_{j}, \omega\right)\right]}{\Im\left[G_{z z}\left(X_{0}, X_{0}, \omega\right)\right]}$

and it is expected to analyze the lateral heterogeneity between two sites.

If we apply Eq. (5) to SPAC method in the simplest equilateral-triangle case, for example, three ratios $\frac{S_{11}(\omega)}{S_{00}(\omega)}, \frac{S_{22}(\omega)}{S_{00}(\omega)}, \frac{S_{33}(\omega)}{S_{00}(\omega)}$ can be obtained to analyze the lateral heterogeneity. Moreover, it is convenient and efficient to calculate this ratio because the power spectra is just the intermediate result in the process of SPAC method. Of course, this concept is not limited to SPAC method but available to any pair of sites in the diffuse wavefield.

\section{The influence of lateral heterogeneity on the ratio of IOG}

It has been mentioned before that IOG indicates intrinsic property of ground structure. It is also the reason

Table 1 The parameters of the two-layered medium

\begin{tabular}{|c|c|c|c|c|}
\hline Layer & $\begin{array}{l}\text { P-wave velocity } \\
(\mathrm{m} / \mathrm{s})\end{array}$ & $\begin{array}{l}\text { S-wave velocity } \\
(\mathrm{m} / \mathrm{s})\end{array}$ & $\begin{array}{l}\text { Density } \\
\left(\mathrm{t} / \mathrm{m}^{3}\right)\end{array}$ & $\begin{array}{l}\text { Thickness } \\
\text { (m) }\end{array}$ \\
\hline 1 & 800 & 400 & 1.5 & 720 to 1280 \\
\hline 2 & 1800 & 1200 & 2.0 & $\infty$ \\
\hline
\end{tabular}

why we want to use the ratio of them to detect the lateral heterogeneity. Hence, it is indispensable to consider the influence of lateral heterogeneity on the ratio of IOG in the first place. In this article, we consider the simplest case of lateral heterogeneity: unidirectional inclined two-layered medium (2D base model). We use FDM to simulate this model (Pitarka 1999).

Before doing that, we examine the availability of the code base on FDM by carrying out a small test as shown in Fig. 2. The simplest two-layered medium with no lateral heterogeneity is simulated using $200 \times 200 \times 50$ meshes with mesh size of $320 \mathrm{~m} \times 320 \mathrm{~m} \times 320 \mathrm{~m}$ in the first layer and $320 \mathrm{~m} \times 320 \mathrm{~m} \times 640 \mathrm{~m}$ in the second layer. In order to demonstrate the layered velocity structure, nonuniform grids are used as shown in Fig. 2. Besides, nonreflecting boundary condition is used to avoid

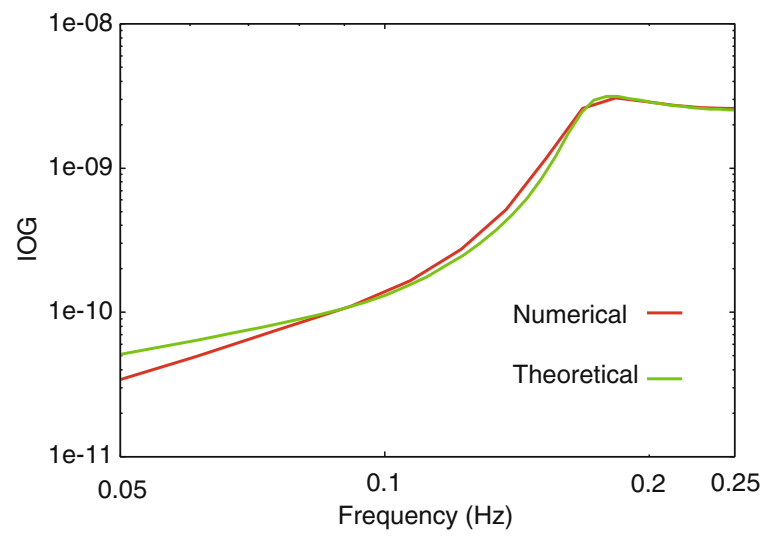

Fig. 3 Imaginary part of Green's function from numerical simulation and theoretical calculation. The $\mathrm{x}$ axis (frequency) is in logarithmic scale 
the reflecting wave (20 meshes wide at boundary) (Charles et al. 1985). Quite large size of mesh size is used here in order to simulate as large area as possible to mitigate the effect of reflecting of long-wavelength wave. The time interval is set to be $0.008 \mathrm{~s}$ and the cutoff frequency is $0.25 \mathrm{~Hz}$ (The higher frequency part is cutted off) (Levander 1988). The duration for simulation is $130 \mathrm{~s}$. The parameters of medium are shown in Table 1. Site A is located at the center of surface as the test site. A vertical unit force is exerted on A so the response at A should be equivalent to $G_{z z}\left(X_{A}, X_{A}\right)$ according to the definition of Green's function. Then, the theoretical Green's function is calculated using technique from (Hisada 1994; 1995). Their imaginary parts match well with each other when $f>0.09 \mathrm{~Hz}$ (Fig. 3). It is believed that by taking ratios, the discrepancy can be neglected. Hence, we can say this indicates the availability of this code based on FDM.

Then, we will use this code to simulate the unidirectionally inclined two-layered medium. The profile is shown in Fig. 4. The related parameters are the same as shown in Table 1. As Fig. 4a shows, eight sites, A to $\mathrm{H}$, are located on the surface with even intervals in the direction of inclination. One site by one site, a vertical unit force is exerted and the $G_{z z}\left(X_{\chi}, X_{\chi}\right)$ $(\chi=\mathrm{A}$ to $\mathrm{H})$ is obtained. The first layer's thickness of each site is shown in Table 2. As described before, the mesh size is set to be quite large so that the inclination can only be simulated roughly. For example, the
Table 2 The first layer's thickness at each site

\begin{tabular}{llllllllll} 
Site name & A & B & C & D & E & F & G & H \\
\hline
\end{tabular}

Equivalent thickness (m) 7208008809601040112012001280

first layer's thickness under site $\mathrm{H}$ and $\mathrm{G}$ is actually the same (Fig. 4b). For convenience, we calculated the equivalent thickness of each site using the linear interpolation as shown in Fig. 4b.

If it is horizontally layered medium, of course the ratios of IOG between any pair of sites are to be 1 . In a complicated 3D medium, this ratio is expected to depend on the particular condition of lateral heterogeneity between two sites. In this simple case of unidirectionally inclined layered medium, the ratio should depend on the difference of first layer's thickness if one of the sites is fixed. In order to observe this, we take ratio of IOG between $\chi$ and $A$ which is $\frac{\Im\left[G_{z z}\left(X_{\chi}, X_{\chi}, \omega\right)\right]}{\Im\left[G_{z z}\left(X_{A}, X_{A}, \omega\right)\right]}(\chi=\mathrm{B}$ to $\mathrm{H})$. The ratios are plotted in Fig. 5 (the solid line). Due to the resolution limit of the numerical simulation, there are some ratios with the same value (for example, $\frac{I O G(H)}{I O G(A)}$ and $\frac{I O G(G)}{I O G(A)}$ ). Despite this minor defect, it is observed that as the difference of first layer's thickness becomes larger, the peak frequency (the frequency where the ratio reaches maximum) shifts to smaller value and the amplitude shifts to larger value.

In a word, in a unidirectionally inclined twolayered medium, there is a peak frequency in the
Fig. 4 The profile of the unidirectionally inclined two-layered medium for calculating Green's function: a eight sites of A to $\mathrm{H}$ in a line with different first layer's thickness. b The cross section of the first layer: the stair shape of the mesh arrangement to roughly simulate the inclination; the mesh number and height at each stair

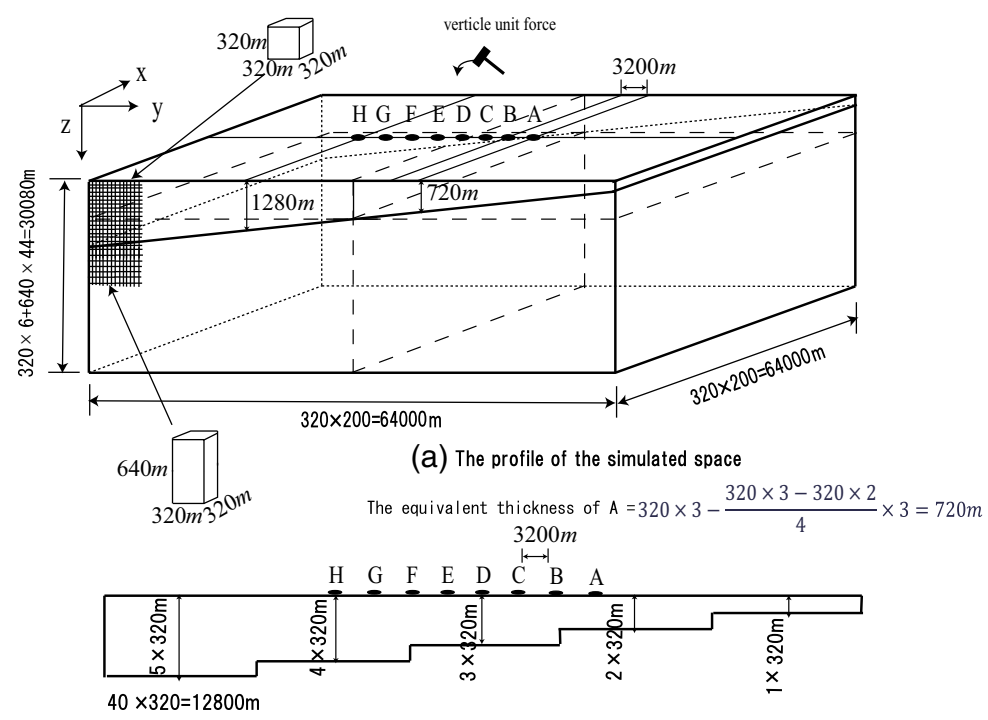

(b) The real condition of the mesh setting in the first layer from a cross section view 


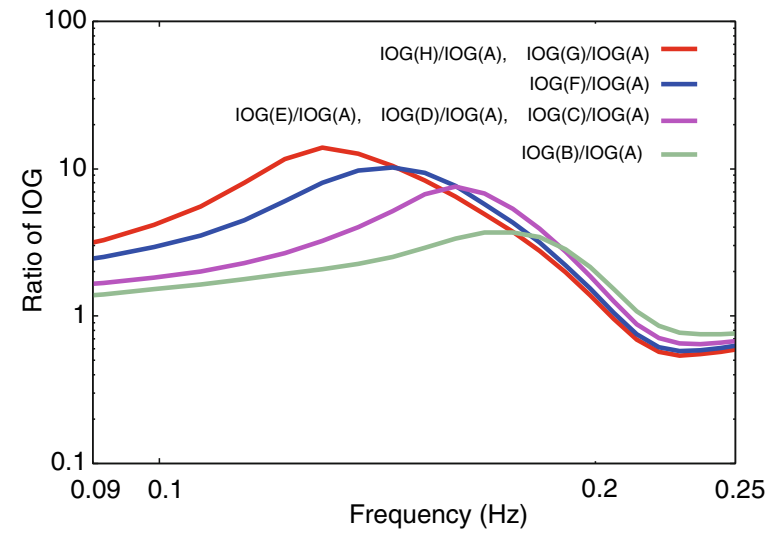

Fig. 5 Ratios of IOG between site $\chi$ and A ( $\chi$ indicates B to $H)$ : the peak frequency shifts to smaller value as $\chi$ moves from $\mathrm{B}$ to $\mathrm{H}$; some ratios overlap due to the limited resolution of numerical simulation (relatively large mesh size). The $\mathrm{x}$ axis (frequency) is in logarithmic scale

ratio of IOG, which depends on the difference of first layer's thickness between two sites if one of the sites is fixed. This forms the theoretical base of making use of ratio of IOG to detect the lateral heterogeneity.

\section{A numerical simulation}

In this chapter, the same FDM code is used to create a diffuse wavefield in the same model as above (Fig. 6).
In this wavefield, the validity of Eq. 5 will be examined among the observation sites.

\subsection{Observation sites}

The observation sites are set in a line (A to $\mathrm{H}$ ), which is the same as the previous section. The concept of vertical component spectral ratio is not limited to any array shape but available at any any pair of sites. Even though the sites are arranged in a line just for simplicity, the simulation does not lose generality for any array methods.

\subsection{To create a diffuse wavefield}

In order to create a diffuse wavefield, we refer to the noise synthetics generation measure in the article by Cornou et al. (2013). Point sources of shear dislocation type with random strike, dip, rake angles, and seismic moment are exerted on mesh at random position of $(x, y, z)(\mathrm{x}, \mathrm{y}$, and $\mathrm{z}$ are mesh number in three directions) using stress approach (Coutant et al. 1995). The position coordinate $(x, y, z)$, angles and the magnitude is randomly produced from a uniform distribution of $\mathrm{U}(0, b)$, in which $b$ is the certain value for different parameters. For example, for $x, b$ is 200 because $x$ varies from 0 to 200. For dip angles, $b$ is $90^{\circ}$. The number of sources is about 1250 per $10 \mathrm{~s}$. Different source number and ways of setting the sources may have different effect on the simulation
Fig. 6 The profile of the unidirectionally inclined two-layered medium for simulating a diffuse wavefield: the eight sites in a line is the same as in the last simulation; point sources of shear dislocation type are produced at random time and with random direction and magnitude to create a diffuse wavefield

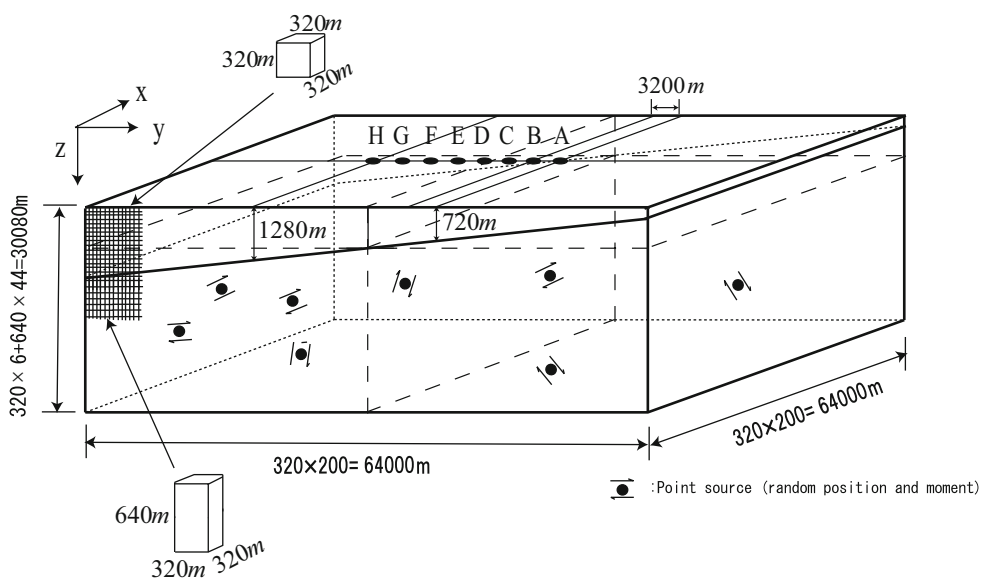


results. We choose this way because it randomize all the parameters of the sources to fit for the actual microtremor wavefield and realize the diffusiveness. The deeper research about the effect on simulation by source settings remains a future topic and let us focus on the result from this simulation.

\subsection{Examination on the new perspective of vertical component ratio of power spectra}

Then, we observe the behavior of the vertical component ratio of power spectra to see whether it coincides with that of ratio of imaginary part of Green's function. For simplicity, we take ratios between $\chi$ and $A$ $(\chi=\mathrm{B}$ to $\mathrm{H})$ in which $A$ works as a reference site (similar with the previous section) and the other seven sites are with different first layer's thickness. Power spectra for each site is calculated (Fig. 7) and the ratios are obtained quickly. The theoretical ratios of IOG, respectively, are calculated, using the same technique as in the previous section, from synthetic waveforms obtained by a 3D numerical simulation with a same observation direction as a dip direction. The result is shown in Fig. 8. The solid lines indicate the ratios of power spectra while different colors indicate different pairs of sites. Compared with the ratios in Fig. 5, it is observed that the amplitude do not match. Moreover, there is the large fluctuation of the amplitude in Fig. 8 among ratios. This comes from the fluctuation of power spectra at each site (Fig 7). Firstly, The different amplification factor at different site (they have

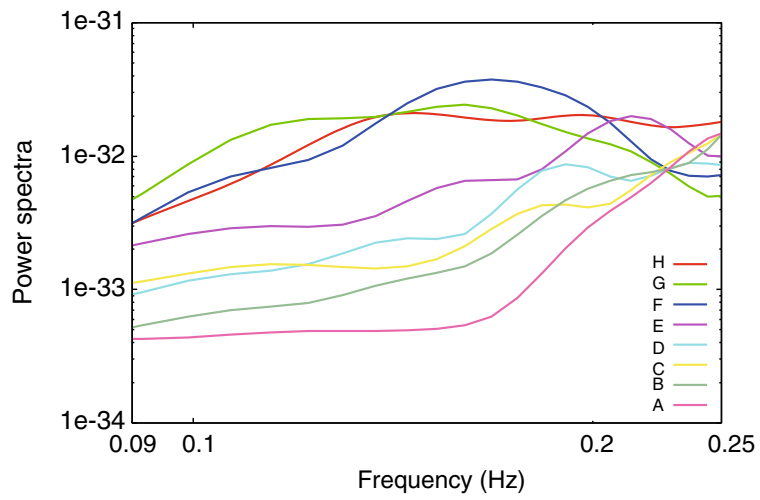

Fig. 7 Power spectra of vertical component at site A to $\mathrm{H}$. The $\mathrm{x}$ axis (frequency) is in logarithmic scale

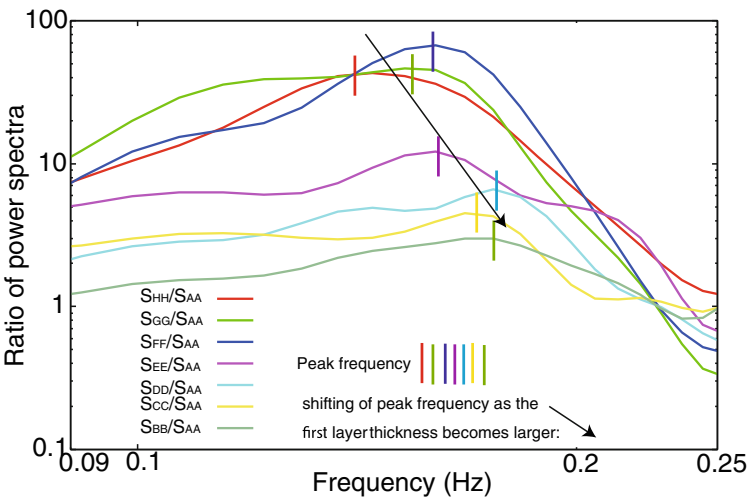

Fig. 8 Ratio of power spectra of vertical component between site $\chi$ and $\mathrm{A}$ ( $\chi$ indicates $\mathrm{B}$ to $\mathrm{H}$ ): the peak frequency is marked by vertical line; the black arrow indicates the shifting of peak frequency as $\chi$ moves from $\mathrm{H}$ to $\mathrm{B}$. The $\mathrm{x}$ axis (frequency) is in logarithmic scale

different first layer's thickness) is considered to be partly the cause. The limited accuracy of numerical simulation is the second cause. Then, we analyze the consistency of the peak frequency from two kinds of ratios between each pari of sites (Fig. 9). The $f_{p(I O G)}$ indicates the peak frequency in the ratio of IOG and the $f_{p(p w)}$ indicates the peak frequency in the ratio of power spectra. It is observed that they match quite

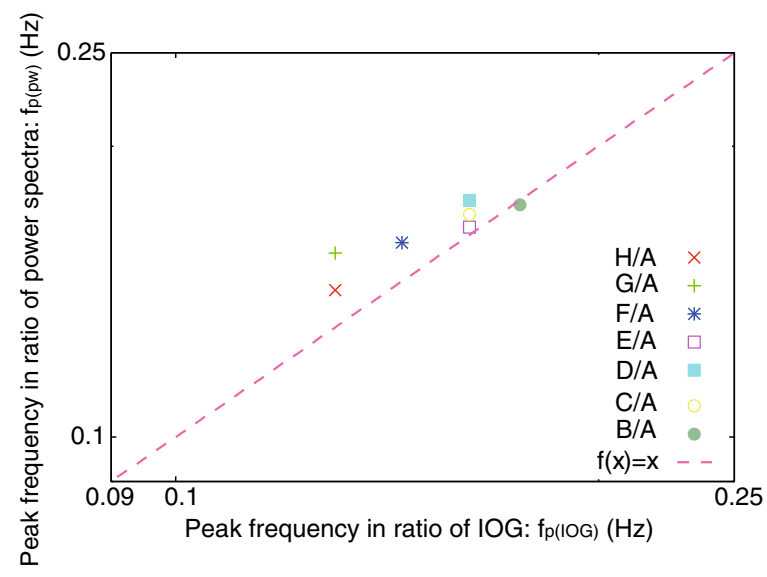

Fig. 9 The consistency check of the peak frequency from two kinds of ratios: the $f_{p(I O G)}$ indicates the peak frequency in the ratio of IOG and the $f_{p(p w)}$ indicates the peak frequency in the ratio of power spectra; different symbol indicates this comparison between different pair of sites; the $f(x)=x$ line helps to see the status of consistency clearly 
well. There exist some discrepancy between the peak frequency from two kind of ratios. Firstly, this may be caused by the resolution limit of the numerical simulation (too big mesh size relative to the site intervals). Secondly, the wavefield is of course not perfectly a diffuse wavefield, which would have bad influence on the result. The setting of source type and source number remain a future work. Neglecting this, it is more meaningful to observe that the peak frequency of ratio decreases as the $\chi$ becomes farther from $A$ (Fig. 8). This tendency coincides with that in Fig. 5. In a word, this result confirms the availability of Eq. (5) in this simulated wavefield.

Combining Figs. 7, 8, and 9, it is observed that by taking vertical component ratio of power spectra between two sites, a peak frequency can be obtained which actually comes from the ratio of IOG between two sites. As for this unidirectionally inclined twolayered medium, the peak frequency changes as the difference of first layer's thickness changes if one of the sites is fixed. These suggest that by applying the vertical component ratio of power spectra between two sites, it is possible to detect and even estimate the lateral heterogeneity (in this case, the inclinaton) in the arrays' area using certain inversion techniques of theoretical Green's function.

\subsection{Discussion}

The availability and the potential of the vertical component spectral ratio between two sites have been demonstrated above. However, as mentioned in the section of introduction, the idea of taking spectral ratio between two sites is not new. The significance of the proposed perspective may be underestimated. In this part, some typical and old concepts about the spectral ratios between two sites would be reviewed. Then, we examine their availability to detect the lateral heterogeneity in the 2D model in the simulated diffuse wavefield.

By taking the horizontal spectral ratio between one site and the reference site, the S-wave resonance frequency can be obtained if the motion at the reference site is representative of the excitation arriving at the interface of soil layer and base (Kagami et al. 1986). It is clear that this concept can not be applied to interpret the ratios between two sites in our numerical simulation, because both sites are on the same surface. On the other hand, in Kanai's theory (Kanai and Tanaka
1954), the ideal 1D base model is assumed for each site (horizontally layered medium) and the horizontal component Fourier amplitude itself is representative of the transfer function of SH wave. Even this concept is based on 1D model and simply vertically $\mathrm{S}$-wave wavefield, it may be doubted that the spectral ratio based on Kanai's theory is enough to detect the lateral heterogeneity in 2D model and full-wave wavefield. Therefore, although Kanai did not apply this idea to the ratio between two sites, we would like to carry this classic concept out for confirmation. Accordingly, the peak frequency obtained from horizontal component ratio of power spectra between two sites is equivalent to the ratio of SH wave transfer function between two sites. In order to examine whether this concept works in this numerical simulation, we calculate the

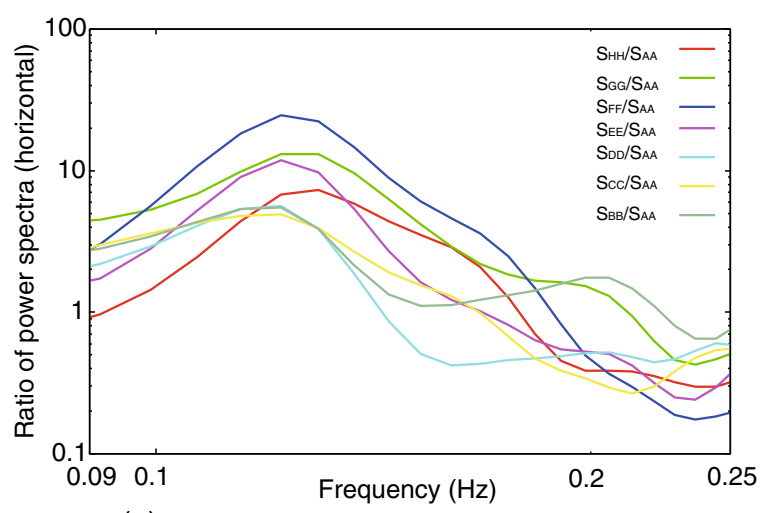

(a) The ratios of power spectra (horizontal component) among sites

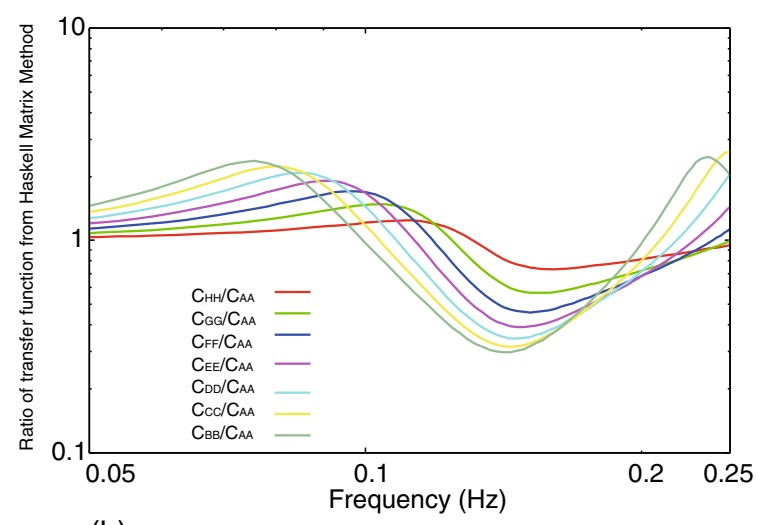

(b) The ratios of transfer function from Haskell Matrix Method among sites

Fig. 10 Ratio about horizontal component between site $\chi$ and A $(\chi$ indicates $B$ to $H)$ : a ratio of power spectra of horizontal component: the peak frequency almost does not shift as $\chi$ moves from $\mathrm{H}$ to $\mathrm{B}(b)$ ratio of transfer function from Haskell Matrix Method: $C_{\chi \chi}$ indicates the transfer function at site $\chi$; peak frequency shifts quickly as $\chi$ moves from $\mathrm{H}$ to $\mathrm{B}$. The $\mathrm{x}$ axis (frequency) is in logarithmic scale 
transfer function of SH wave using Haskell Matrix Method for each site and take the ratios between the same seven pairs of sites as in previous subsection. The comparison with the horizontal component ratio of power spectra is shown in Fig. 10. Figure 10a shows the ratio of power spectra of horizontal component and Fig. 10b shows the ratio of transfer function from Haskell Matrix Method. $C_{\chi \chi}$ indicates the transfer function from Haskell Matrix Method at site $\chi(\chi$ is A to H). From the comparison between Fig. 10a and $\mathrm{b}$ about the peak frequency of the same color curve, it is obvious that the peak frequency from two kinds of ratios are totally different. The peak frequency from the ratio of power spectra of horizontal component (Fig. 10a) is obviously larger than the ratio of transfer function from Haskell Matrix Method (Fig. 10b). For instance, let us compare the peak frequency of the blue curve (D/A); the peak frequency in (a) is obviously larger than 0.1 while it is smaller than 0.1 in (b).

To be more exact, the peak frequency from two kinds of ratios are compared in Fig. 11. The $f_{p(h v)}$ indicates the peak frequency in the ratio of transfer function from Haskell Matrix Method and the $f_{p(p w h)}$ indicates the peak frequency in the horizontal component ratio of power spectra. It is obvious that the two ratios deviate from each other very much.

This result suggests that in this numerical simulation, the horizontal component ratio of power spectra

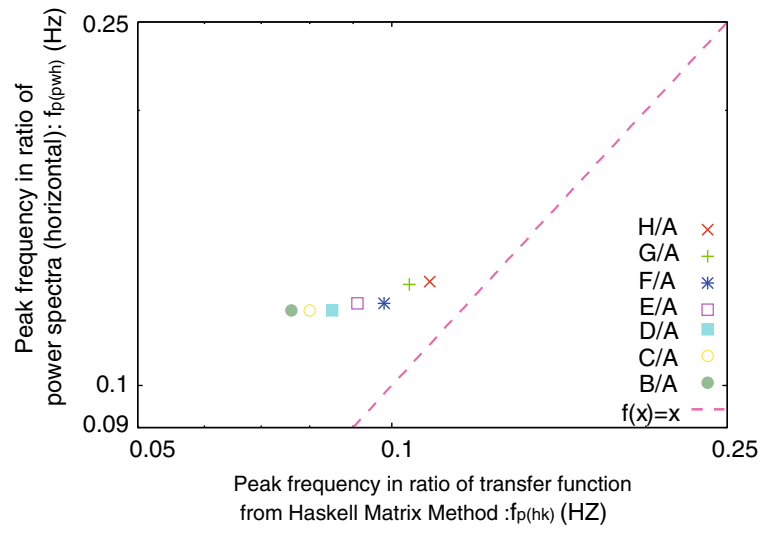

Fig. 11 The consistency check of the peak frequency from two kinds of ratios: the $f_{p(h v)}$ indicates the peak frequency in the ratio of transfer function from Haskell Matrix Method and the $f_{p(p w h)}$ indicates the peak frequency in the horizontal component ratio of power spectra; different symbol indicates this comparison between different pair of sites; the $f(x)=x$ line helps to see the status of consistency clearly between two sites cannot be explained by simply the ratio of transfer function obtained from 1D base model well. It is because in Kanai's theory, the 2D base model and the condition of full wave are not taken into consideration. The conventional method is of course available in a $1 \mathrm{D}$ model. However, it is meaningful to say that in a complicated base model, it is better to use the Green's function theory based on the assumption of full wave to explain the ratio of power spectra between two sites. In this article, the vertical component ratio of power spectra between two sites in a 2D base model is successfully interpreted using the IOG. In order to avoid misunderstanding, we here emphasize that we are not saying using horizontal component spectral ratio is inferior than using vertical component spectral ratio. We are stressing the unavailability of using the ratios based on 1D model to explain the ratios from actual records in 3D medium, no matter it is horizontal component or vertical component.

Moreover, $f_{p(p w h)}(\chi A)$ almost does not change as the site $\chi$ moves from $H$ to $B$. This means $f_{p(p w h)}$ has no sensitivity to the lateral heterogeneity so it is difficult to use it to estimate ground structure. However, this is not the point of

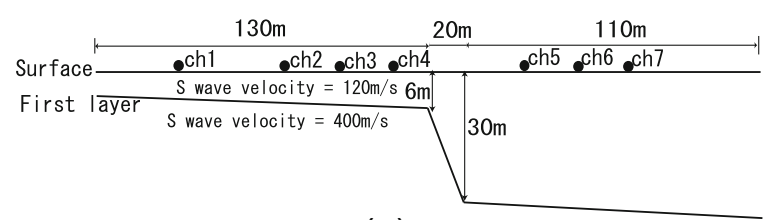

(a)

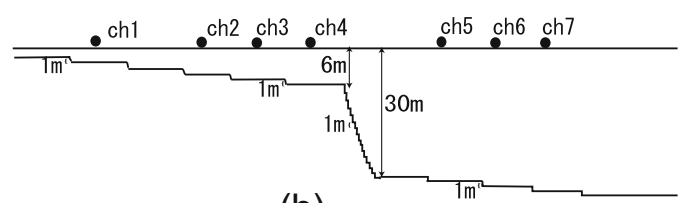

(b)

Fig. 12 The soil profile of the area and its simulation: a The rough profile according to the boring data. There is a steep inclination in the middle part and seven sensors set on the surface, the S-wave velocity of both layers are shown. b The simulation model of the rough structure in (a) is demonstrated. We use mesh size of $1 \mathrm{~m}$ and simulate the inclination by setting different mesh number in the depth direction at each site. Linear inclination is used. For the steep inclination in the middle part, the first layer's thickness varies about $30-6=24 \mathrm{~m}$ in a horizontal distance of $20 \mathrm{~m}$. For the left and right part, a very mild inclination is assumed. The first layer's thickness varies about $1 \mathrm{~m}$ in a horizontal distance of $25 \mathrm{~m}$ 
this article, which remains to be discussed in the future.

\section{A field test}

To confirm more about the relationship of the two ratios, let us see a field test conducted in Aomori, Japan, in which we have seven sensors $\operatorname{ch} 1$ to $\operatorname{ch} 7$ recording the microtremor waves (Sakai et al. 2015). According to the boring data, the profile of the observation area is shown as Fig. 12. In Fig. 12a, the positions of sensors and the soil profile are shown. It can be seen that there is a very steep inclination of the first layer, in which the first layer's thickness varies about $30-6=24 \mathrm{~m}$ in a horizontal distance of
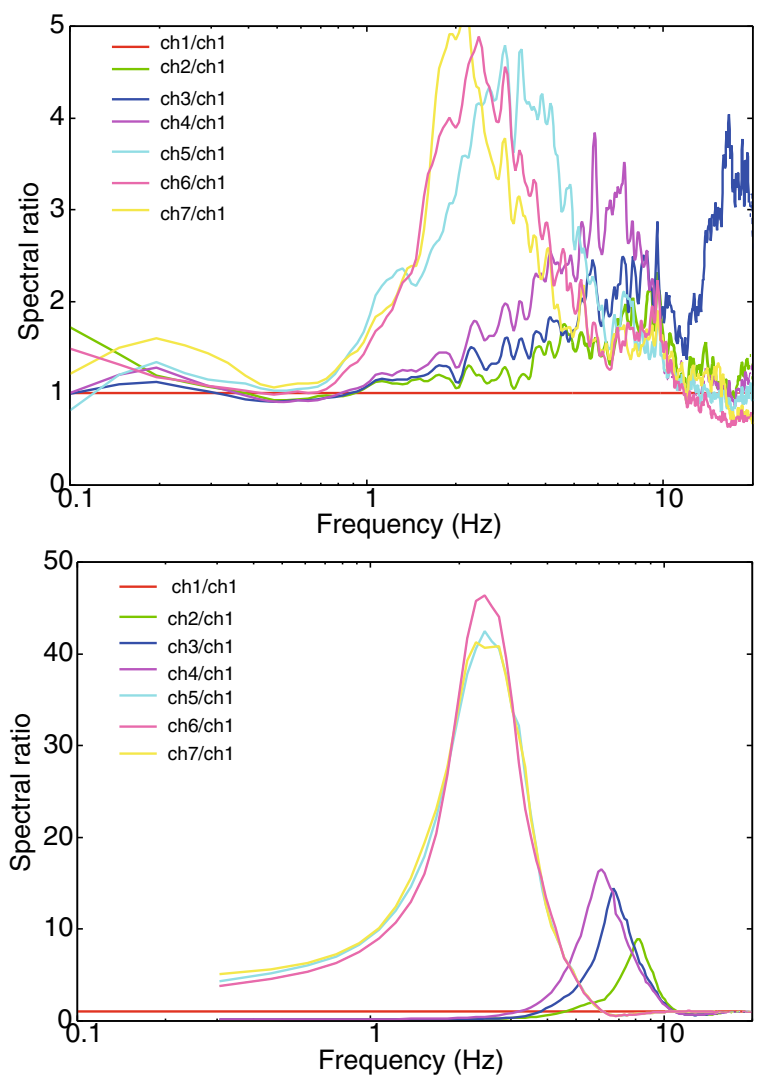

Fig. 13 Ratio of IOG and power spectra between site $\chi$ and $\operatorname{ch} 1$ ( $\chi$ indicates $\operatorname{ch} 2$ to $\operatorname{ch} 7$ ): a ratio of power spectra recorded from the field test, and $\mathbf{b}$ ratio of IOG obtained from numerical simulation. It can be observed that the peak frequency of both the ratios shifts to smaller value as the $\chi$ site shifts from $\operatorname{ch} 2$ to $\operatorname{ch} 7$
$20 \mathrm{~m}$. Left to the inclination, it is a shallow $\mathrm{n}$ on which sensors $\operatorname{ch} 1, \operatorname{ch} 2, \operatorname{ch} 3$, and $\operatorname{ch} 4$ are set. There is a relatively large interval between $c h 1$ and $c h 2$. Right to the inclination, it is a deep structure on which sensors ch5, ch6, ch 7 are set. The structures of both sides may have very mild inclination.

In order to obtain the theoretical IOG at the position of each site, a numerical simulation is done. According to the rough structure from boring data, we simulate the structure using mesh size of $1 \mathrm{~m} \times 1 \mathrm{~m} \times 1 \mathrm{~m}$ as shown in Fig. 12b. For the simulation of the steep inclination, we assume a linear inclination, in which the first layer's thickness varies about $30-6=24 \mathrm{~m}$ in a horizontal distance of $20 \mathrm{~m}$. For the simulation of structure left and right to the steep inclination, we assume a very mild linear inclination, in which the first layer's thickness varies $1 \mathrm{~m}$ in a horizontal distance of $25 \mathrm{~m}$. By exerting a unit vertical force at each site and recording the response at that site, the IOG at each site can be obtained.

The ratio of power spectra of vertical component between $\chi$ and $\operatorname{ch} 1,(\chi=\operatorname{ch} 2$ to $\operatorname{ch} 7)$ and the ratio of the IOGs are shown in Fig. 13. Figure 13a shows ratio of power spectra recorded from the field test with different colors. Figure $13 \mathrm{~b}$ shows the ratio of IOG obtained from numerical simulation. It can be observed that the peak frequency of both the ratios shifts to smaller value as the $\chi$ site shifts from $\operatorname{ch} 2$ to

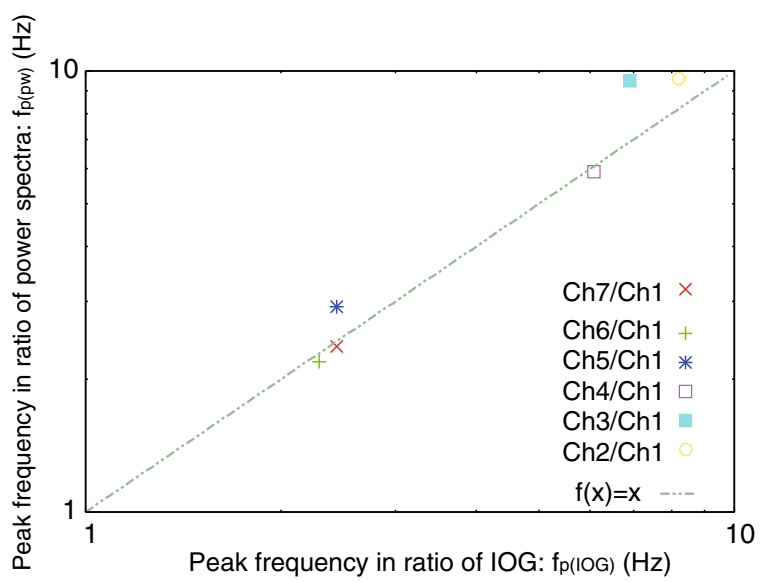

Fig. 14 The consistency check of the peak frequency from two kinds of ratios: the $f_{p(I O G)}$ indicates the peak frequency in the ratio of IOG and the $f_{p(p w)}$ indicates the peak frequency in the ratio of power spectra; different symbol indicates this comparison between different pair of sites; the $f(x)=x$ line helps to see the status of consistency clearly 
$c h 7$. It is observed that the ratio of IOG for one pair of sites has a peak frequency which is similar to that from the ratio of power spectra for the same pair of sites. For the $\operatorname{ch} 3 /$ ch 1 ratio in Fig. 13a, it has spikes above $10 \mathrm{~Hz}$, which cannot be explained by the ratios of IOG in the simulated model. We consider it is most likely from the noise in high-frequency range such as the traffic noise and has no influence on this test. It is also possibly from the influence of the real ground structure. It remains to be discussed in the future. However, this does not affect the fundamental objective of this field test.

The consistency of the peak frequency from two kinds of ratios between each pari of sites is shown in Fig. 14. The $f_{p(I O G)}$ indicates the peak frequency in the ratio of IOG and the $f_{p(p w)}$ indicates the peak frequency in the ratio of power spectra. It is observed that they match quite well. It is confirmed that, even in a field test using the actual data, the peak frequency from the ratio of power spectra between two sites matches with the ratio of IOG between the same two sites.

\section{Conclusion}

Using seismic interferometry theory, we propose a new perspective of the vertical component ratio of power spectra between two sites in diffuse wavefield. Through theoretical analysis, a numerical simulation and a field test, there are several conclusions to be drawn.

1. There exists a peak frequency in the vertical component ratio of power spectra between two sites. It depends on the particular condition of lateral heterogeneity between two sites.

2. In a simple unidirectionally inclined two-layered medium in diffuse wavefield, the peak frequency depends simply on the difference of first layer's thickness and the average thickness of first layer. As for a ratio $\frac{S_{x x}}{S_{a a}}$ (first thickness of site $x$ bigger than that of site $a$ ) with the site $a$ fixed, the peak frequency decreases as the site $x$ becomes farther.

3. In an inclined two-layered medium, by taking the vertical component ratios of power spectra among sites at arrays, the peak frequency can be a good indicator of lateral heterogeneity by applying the equivalence with that from the ratio of IOG.
4. In this numerical simulation, it does not work well to interpret the horizontal component ratio of power spectra using the ratio of transfer function of SH wave based on 1D model. Hence, it demonstrates that in the condition of full wave and in complicated base model, it is better to use Green's function theory to interpret the spectral ratio between two sites.

This article demonstrates a new interpretation of the vertical component ratio of power spectra and demonstrates its potential of detecting lateral heterogeneity in a simple numerical simulation and a field test using the peak frequency. It remains as a future work to develop the inversion technique to estimate the particular condition of lateral heterogeneity from the peak frequency among sites in a 2D base model or more complicated model.

\section{References}

Aki K (1957) Space and time spectra of stationary stochastic waves, with special reference to microtremors. Bull Earthquake Res Inst 25:415-457

Campillo M, Paul A (2003) Long range correlations in the diffuse seismic coda. Science 299:547-549

Charles C, Kosloff D, Kosloff R, Reshef M (1985) A nonreflecting boundary condition for discrete acoustic and elastic wave equations. Geophysics 50:705-708

Chávez-García FJ, Rodrígues M, Stephenson WR (2005) An alternative approach to the SPAC method analysis of microtremors: exploiting stationarity of noise. Bull Seism Soc Am 95:277-293

Chávez-García FJ, Rodrígues M, Stephenson WR (2007) Subsoil structure using SPAC measurement along a line. Bull Seism Soc Am 96:729-736

Cho I, Tada T, Shinozaki Y (2006) A generic formulation for microtremor exploration method using three-component records from a circular array. Geophys J Int 165:236-258

Claerbout JF (1968) Synthesis of a layered medium from its acoustic transmission response. Geophysics 33:264-269

Cornou C, Ohrnberger M, Boore DM, Kudo K, Bard P-Y (2013) Derivation of structural models from ambient vibration array recordings: results from an international blind test. J Softw 8(3):666-672

Coutant O, Virieux J, Zollo A (1995) Numerical source implementation in a $2 \mathrm{D}$ finite difference scheme for propagation. Bull Seism Soc Am 85:1507-1512

Hisada Y (1994) Efficient method for computing green's functions for a layered half-space with sources and receivers at close depths. Bull Seism Soc Am 84(5):1456

Hisada Y (1995) Efficient method for computing green's functions for a layered half-space with sources and receivers at close depths (Part 2). Bull Seism Soc Am 85(4):1080 
Kagami H, Okada S, Shiono K, Oner M, Dravinski M, Mal AK (1986) Observation of 1 to 5 second microtremors and their application to earthquake engineering. Part III. A twodimensional study of site effects in S. Femando valley. Bull Earthquake Res Inst 76:1801-1812

Kanai K, Tanaka T (1954) Measurement of the microtremor. Bull Earthquake Res Inst 32:199-209

Kawase H, Sánchez-Sesma FJ, Matsushima S (2011) The optimal use of horizontal-to-vertical ratios of earthquake motions for velocity inversions based on diffuse field theory for plane wave. Bull Seism Soc Am 101(5):2001-2014

Lermo J, Chávez-García FJ, Matsushima S (1994) Are microtremors useful in site response evaluation? Bull Seism Soc Am 84(5):1350-1364

Levander AR (1988) Fourth-order finite-difference P-SV seismograms. Geophysics 53:1425-1436

Morikawa H, Sawada S, Akamatsu J (2004) A method to estimate phase velocity of Rayleigh waves using microtremors simultaneously observed at two sites. Bull Seism Soc Am 94:961-976

Okada H (2003) Microtremor survey method. SEG Geophysical Monograph Series No 12

Paul A, Campillo M, Margerin L, Larose E, Derode A (2005) Empirical synthesis of time-asymmetrical Green function from the correlation of coda waves. J Geophys Res 110. doi:10.1039/2004JB003521

Perton M, SanchezSesma FJ, Rodriguez-Castellanos A, Campillo M, Weaver RL (2009) Two perspectives on equipartition in diffuse elastic fields in three dimensions. $\mathbf{J}$ Acoust Soc Am 126:1125-1130

Pitarka A (1999) 3D elastic finite-difference modeling of seismic motion using staggered grids with nonuniform spacing. Bull Earthquake Res Inst 89:54-68

Sakai K, Tanaka K, Usami A, Kobayashi K, Hirabayashi M (2015) A study for detection of the site on inclined engineering bedrock based on microtremor observation. In: Japan Geoscience Union Meeting, Makuhari Messe, Japan, vol 89, pp 54-68

Sánchez-Sesma FJ, Campillo M (2006) Retrieval of the Green's function from cross correlation: the canonical problem. Bull Seism Soc Am 96(3):1182-1191

Sánchez-Sesma FJ, Perez-Ruiz JA, Campillo M, Luzon F (2006) Elastodynamic 2D Green's function retrieval from cross-correlation: canonical inclusion problem. Geophys Res Lett 33:L13305

Sánchez-Sesma FJ, Pérez-Ruiz JA, Luzón F, Campillo M (2008) Diffuse fields in dynamic elasticity. Wave Motion 45:641-654

Sánchez-Sesma FJ (2011) A theory for microtremor H/V spectral ratio: application for a layered medium. Geophys J Int 186(1):221-225

Shiraishi H, Matsuoka T, Asanuma H (2006) Direct estimation of the Rayleigh wave phase velocity in microtremors. Geophys Res Lett 33:L18307

Snieder R (2004) Extracting the Green's function from the correlation of coda waves: a derivation based on stationary phase. Phys Rev E69:254301

Tada T, Cho I, Shinozaki Y (2009) New circular-array microtremor techniques to infer Love-wave phase velocity. Bull Seismol Soc Am 99(5):2912-2926

Tsai VC, Moschetti MP (2010) An explicit relationship between time-domain noise correlation and spatial autocorrelation (SPAC) results. Geophys J int 172(1):293-301

Wapenaar K, Draganov D, Thorbecke J, Forkkema J (2002) Theory of acoustic daylight imaging revisited. In: 72nd Annual International Meeting, SEG Expanded Abstracts, pp 2269-2272

Wapenaar K (2004) Retrieving the elastodynamic Green's function of an arbitrary inhomogeneous medium by cross correlation. Phys Rev Lett 93:254301

Wapenaar K, Thorbecke J, Draganov D (2004) Relations between reflection and transmission responses of threedimensional inhomogeneous media. Geophys $\mathrm{J}$ Int 156:179-194

Wapenaar K, Draganov D, Robertson J (2006) Introduction to the supplement on seismic interferometry. Geophysics 71:SI1-SI14

Wapenaar K, Fokkema J (2006) Green's function representations for seismic interferometry. Geophysics 71:SI33SI46

Weaver RL, Lobkis OI (2001) Ultrasonics without a source: Thermak fluctuation correlations at $\mathrm{MHz}$ frequencies. Phys Rev Lett 87:134301

Yokoi T, Margaryan S (2008) Consistency of the spatial autocorrelation method with seismic interferometry and its consequence. Geophys Prospect 56:435-451 